\title{
ELASTIC STRESSES DUE TO A SEMI-INFINITE BAND OF HYDROSTATIC PRESSURE ACTING OVER A CYLINDRICAL HOLE IN AN INFINITE SOLID*
}

\section{By O. L. BOWIE (Watertown Arsenal, Watertown, Mass.)}

Recently C. J. Tranter ${ }^{1}$ has presented expressions for the stresses due to a finite band of hydrostatic pressure acting over part of the length of a cylindrical hole which extends through an infinite elastic solid. By a similar analysis, expressions for the stresses due to a semi-infinite band of hydrostatic pressure acting over the cylindrical hole can be found. Referring to the analysis by Tranter, it is evident that the only change in boundary conditions is that involving $\sigma_{r}$ at $r=a$.

A semi-infinite band of hydrostatic pressure, $p$, is equivalent to the sum of

$$
\sigma_{r}=-\frac{p}{2}, \quad\left\{\begin{array}{c}
-\infty<z<\infty \\
r=a
\end{array}\right.
$$

and

$$
\left.\begin{array}{rrr}
\sigma_{r} & =-\frac{p}{2}, & -\infty<z<0 \\
=\frac{p}{2}, & 0<z<\infty
\end{array}\right\} r=a .
$$

The stresses due to the loading (1) are well known, ${ }^{2}$ whereas, the analysis corresponding to (2) may be carried out in a manner very similar to that used by Tranter. The combined stress distributions determined for (1) and (2) yield the following expressions for the stresses:

$$
\begin{gathered}
\sigma_{r}=-\frac{p}{2}\left(\frac{a^{2}}{r^{2}}\right)-\frac{p a}{\pi r} \int_{0}^{\infty}\left[\alpha \rho K_{0}(\alpha) K_{0}(\rho)+\alpha K_{0}(\alpha) K_{1}(\rho)-\rho K_{0}(\rho) K_{1}(\alpha)\right. \\
\left.-\left\{\rho^{2}+2(1-\nu)\right\} K_{1}(\alpha) K_{1}(\rho)\right] \cdot \frac{1}{\alpha D(\alpha)} \sin (z \alpha / a) d \alpha \\
\tau_{r z}=+\frac{p}{\pi} \int_{0}^{\infty}\left[\alpha K_{0}(\alpha) K_{1}(\rho)-\rho K_{0}(\rho) K_{1}(\alpha)\right] \cdot \frac{1}{D(\alpha)} \cos (z \alpha / a) d \alpha \\
\sigma_{\theta}=\frac{p}{2}\left(\frac{a^{2}}{r^{2}}\right)+\frac{p a}{\pi r} \int_{0}^{\infty}\left[\alpha K_{0}(\alpha) K_{1}(\rho)+(2 \nu-1) \rho K_{0}(\rho) K_{1}(\alpha)\right. \\
\left.\quad-2(1-\nu) K_{1}(\alpha) K_{1}(\rho)\right] \cdot \frac{1}{\alpha D(\alpha)} \sin (z \alpha / a) d \alpha \\
\sigma_{z}=\frac{p}{\pi} \int_{0}^{\infty}\left[\alpha K_{0}(\alpha) K_{0}(\rho)+2 K_{0}(\rho) K_{1}(\alpha)-\rho K_{1}(\alpha) K_{1}(\rho)\right] \cdot \frac{1}{D(\alpha)} \sin (z \alpha / a) d \alpha
\end{gathered}
$$

where the notation is that used by Tranter.

* Received Sept. 27, 1946.

1 C. J. Tranter, On the elastic distortion of a cylindrical hole by a localized hydrostatic pressure, Quart. Appl. Math. 4, 298 (1946).

2 A. H. Love, Theory of elasticity, Cambridge Press, 1927, p. 144. 
It is evident that by a proper superposition of the preceding results, Tranter's expressions for a finite band of pressure may be obtained immediately. A similar variation on Rankin's solution ${ }^{3}$ for the case of a finite band of external pressure acting on a solid cylinder will yield expressions for the stresses corresponding to a semiinfinite band of external radial pressure acting on a solid cylinder.

\title{
ON A CONFORMAL MAPPING TECHNIQUE*
}

\author{
By G. F. CARRIER (Brown University)
}

1. Introduction. Many physical problems are readily reduced to the problem of finding the value of a harmonic function on a smooth closed curve corresponding to given boundary conditions and certain interior singularities. For example, it has been shown [1] that the problem of finding the flow of an incompressible non-viscous fluid past a periodic array of airfoils ${ }^{1}$ may be replaced by that of determining the flow within a smooth closed stream-line $C$ with a source-vortex and a sink-vortex at specified interior points. ${ }^{2}$ Analogous problems can obviously occur in other physical situations which lead to the Laplace equation.

Here we shall develop a method of mapping a smooth closed curve $C$ conformally onto the unit circle so as to carry two arbitrarily specified interior points into the points $\pm a$. The mapping is continuous within and on $C$.

Since the solution requires the integration of a single non-homogeneous Fredholm integral equation, we also present a numerical procedure which is useful in solving such equations. The over-all procedure seems preferable, in general, to Theodorsen's method of mapping "nearly circular regions" [2], [3], since the equation used here has a non-singular kernel in contrast to those in his two simultaneous integral equations.

2. The integral equation. Let us consider the problem of finding that complex potential $F(\zeta)=\phi+i \psi$ on the closed curve $C^{3}$ (see Fig. 1) which corresponds to two equal and opposite interior logarithmic singularities at the points $P$ and $Q$ and let $\psi$ vanish on $C$. We may write then, for points on and within $C$,

$$
F(\zeta)=\ln [(\zeta-P) /(\zeta-Q)]+f(\zeta),
$$

where $f$ is analytic within $C$ and continuous on $C$. In principle, no difficulty, would be encountered if the unit coefficients of these singularities were replaced by complex numbers, but the present form is sufficiently general for our purpose.

Consider the integral

${ }^{3}$ A. W. Rankin, Shrink-fit stresses and deformations, J. Appl. Mech. 11, A-77 (1944).

* Received Sept. 13, 1946.

1 The airfoil shape being arbitrary.

${ }^{2} C$, in this case, is a non-self-intersecting convex curve with a continuously turning tangent. By "smooth" we shall henceforth imply such a curve.

${ }^{8} C$ is any closed, not self-intersecting, curve. 